\title{
Cerebellar haematomas caused by angiomas in children ${ }^{1}$
}

\author{
G. ERENBERG, ${ }^{2}$ R. RUBIN, AND K. SHULMAN
}

From the Saul R. Korey Department of Neurology, the Division of Pediatric Neurosurgery of the Department $\stackrel{3}{\exists}$ of Neurological Surgery, and the Department of Pediatrics, Albert Einstein College of Medicine, Bronx Municipal Hospital Center, and Montefiore Hospital and Medical Center, Bronx, N.Y., U.S.A.

SUMMARY Spontaneous cerebellar haematomas in previously well children are most often caused by haemorrhage from small angiomas. Eight such cases in children 12 years of age or younger have $\mathbb{\nabla}$ been reported previously. Their clinical course was usually not as acute as the course most commonly seen in adults, and four of the children survived after evacuation of the haematoma. Two additional

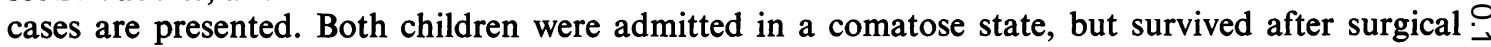
intervention. Cerebellar haematomas in children seem to have a better prognosis than in adults and $\vec{\omega}$ should be considered in the evaluation of children with subarachnoid haemorrhage or the rapid onset of coma. Even if admitted in extremis, recovery is possible after prompt diagnosis and surgical evacuation of the haematoma.

There has been a recent increase of interest in cerebellar haematomas as a surgically correctable neurological lesion. Spontaneous cerebellar haemorrhage as a cause of death was first described in 1813 by Sedillot who also cited prior cases. By 1942 Mitchell and Angrist were able to collect 109 cases from the world literature and added 15 cases from their own series. Large necropsy series have revealed that cerebellar haemorrhages constitute 1 to $15 \%$ of all intracranial haemorrhages (Michael, 1932; Mitchell and Angrist, 1942; Fang and Foley, 1954; ReyBellet, 1960; Dinsdale, 1964). The averaged incidence, in the larger series, points to approximately one patient in 10 with spontaneous intracranial haemorrhage having the primary lesion limited to the cerebellum. This correlates with the fact that the cerebellum accounts for $10 \%$ of the total weight of the brain.

Several studies have reviewed the problems in pre-mortem diagnosis and have listed the various aetiologies (Hyland and Levy, 1954; Rey-Bellet, 1960; Dinsdale, 1964; Norris, Eisen, and Branch, 1969). Cerebellar haematomas occur predomin-

\footnotetext{
${ }^{1}$ This work was supported in part by a Special Traineeship Award number 2F11NB01965, from the National Institute of Neurological Diseases and Stroke.

2 Reprint requests: Gerald Erenberg, Pediatric Neurology, Montefiore Hospital and Medical Center, 111 E. 210 St., Bronx, New York 10467, U.S.A.
}

antly in adults, with hypertension the cause in 59 to $90 \%$ of cases (Rey-Bellet, 1960 ; Dinsdale, 1964 요을 Norris et al., 1969). Angiomas are thought to be the aetiology in the majority of younger patients

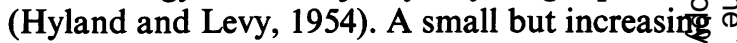
number of operative successes have been ret $\overrightarrow{0}$ ported (Lichtenstein, 1968), but reports of such $N$ successes in children under the age of 12 years have been rare. Recent experience with two young children who were admitted in coma and survived after surgical evacuation of cerebellar haematomas prompted us to review the literature and report these cases in detail.

\section{CASE 1}

R. T. is a 9 year old right-handed boy who was well until 9 January 1970, the day of admission. While playing quietly, he suddenly complained of excruciating occipital headache, ran around the room in a confused state and fell to the floor unresponsive. He was taken to another hospital and arrived there within 15 minutes. When first seen his blood pressure was $110 / 80 \mathrm{~mm} \mathrm{Hg}$, pulse 80 per min. $\rightarrow$ He had agonal respirations, with equal, dilated nonreactive pupils, absent corneal, oculocephalic, and No cold caloric responses, a flaccid quadriplegia, and no withdrawal to noxious stimuli. Nuchal rigidity was not present. There was no papilloedema or fundal $\mathfrak{\omega}$ haemorrhage. A lumbar puncture revealed bloody xanthochromic fluid with an opening pressure of $\leftrightarrow$ 
$450 \mathrm{~mm}$ of water and haematocrit of $8 \%$. After prompt intubation and assisted ventilation he was transferred to Montefiore Hospital and Medical Center (MHMC) where his examination was unchanged except for the presence of occasional withdrawal to noxious stimuli and flexor plantar responses.

DIAGNOSTIC STUDIES AND CLINICAL COURSE Immediately upon arrival at MHMC, left transfemoral vertebral and right carotid arteriograms were performed. These studies demonstrated ventricular dilatation and a left cerebellar haematoma, with a contiguous vascular malformation feeding from the left superior cerebellar artery. Ventricular drainage was instituted and maintained overnight, and intravenous dexamethasone and hypothermia were begun. During this time spontaneous respiration returned, and he developed decerebrate posturing on the left side. Plantar responses became extensor. Corneal and oculocephalic responses remained absent.

SURGERY Eighteen hours after the acute onset of symptoms, a left cerebellar haematoma and an overlying subdural haematoma were evacuated through a suboccipital craniectomy. The cerebellar haematoma occupied the superior and lateral one-half of the left hemisphere. Several abnormal vessels, both arteries and veins, were encountered at the base of the haematoma cavity. The remainder of the posterior fossa, including the fourth ventricle, was free of abnormal vessels. Microscopic sections of the surgical specimen demonstrated an angiomatous malformation containing arterial and venous components.

POSTOPERATIVE COURSE There was some immediate improvement with return of pupillary light responses and occasional spontaneous limb movements. The dominant posture, however, was bilaterally decerebrate. Tracheostomy was required at this time. Oculocephalic and corneal reflexes were absent. The only changes in his neurological status during the next three weeks were the occasional presence of ocular bobbing and an increase in purposeful movements.

Because of a persistently bulging suboccipital decompression, a pneumoencephalogram was performed on the 23 rd postoperative day and revealed mild communicating hydrocephalus. Decerebrate posturing stopped. His state of consciousness improved slightly around this time, but then remained unchanged for the next three months. His eyes were usually open, and he intermittently followed moving objects with his eyes and attempted to follow verbal commands. Observations of his behaviour seemed to indicate that sleep and awake cycles were present. Standard electroencephalograms (EEG) obtained one, two, and three months after surgery revealed symmetrical and diffuse, moderate to high voltage
5-6 Hz activity. Sleep spindles appeared to be present on the first two EEGs. A feeding gastrostomy was performed. A second pneumoencephalogram on the 48th postoperative day revealed increased hydrocephalus, and a ventriculojugular shunt and plastic repair of the pseudomeningocoele were therefore performed.

Five months after the initial surgery, a remarkable recovery began. He became awake and alert, was able to communicate verbally and regained good functional use of his upper extremities. Even now, many months after the apoplectic event, recovery continues to be made. Examination now reveals a decreased left corneal reflex and a slight left peripheral facial weakness. There is a mild left hemiparesis, increased deep tendon reflexes in both lower extremities, and bilateral extensor plantar responses. Horizontal and vertical nystagmus can be elicited. A cerebellar speech pattern is present. Cerebellar signs are present to a moderate degree in the upper and lower extremities on the right side and to a marked degree on those of the left. He is still unable to walk independently, but cares for himself in all other ways. He receives home instruction with a teacher provided by the school system. WISC testing reveals a verbal IQ of 76 . Before his illness he was an average student. Future placement in a class for the physically handicapped is being arranged.

\section{CASE 2}

M. M. is an 8 year old right-handed girl who was well until 20 December 1969, the day of admission, when she noted the sudden onset of a left parietal headache, screamed with pain, felt dizzy, and was noted to have a staggering gait. She was put to bed, and one hour later had a generalized seizure. She was taken to another hospital where she was found to be comatose with absent corneal and oculocephalic responses but with equal, reactive pupils and normal, spontaneous respiration. No papilloedema was noted. Opening lumbar spinal fluid pressure was $300 \mathrm{~mm}$ of water with a haematocrit of $10 \%$ and xanthochromic supernatant fluid.

Six hours after the onset of her symptoms she was transferred to Bronx Municipal Hospital Center (BMHC) where examination revealed a flaccid, unresponsive child with irregular respiration, intermittent spontaneous bilateral decerebrate posturing, vertical nystagmus, absent plantar responses, and ocular bobbing. Cold caloric responses were absent bilaterally. Shortly thereafter the right pupil became 1-2 mm larger than the left and both were unreactive to light. On arrival at BMHC she was intubated and started on intravenous dexamethasone, mannitol, and hypothermia. Assisted respiration was required for the first 24 hours.

DIAGNOSTIC STUDIES AND CLINICAL COURSE Radio- 
graphs of the skull were normal. Percutaneous right carotid angiography was performed within two hours of arrival and showed evidence of slight ventricular enlargement with elevation of the internal cerebral vein. This study was followed by a transfemoral right vertebral arteriogram which was initially interpreted as normal. Subsequent review of the films suggests an avascular mass in the right cerebellar hemisphere. During the next two days the patient improved slightly, with return of corneal reflexes and spontaneous bilateral semi-purposeful movements. Tracheostomy was performed, but the patient maintained spontaneous respiration. A ventriculogram performed two days after admission demonstrated anterior displacement of the aqueduct with enlargement of the lateral and third ventricles. A left vertebral arteriogram was then performed and was interpreted as normal. Ventricular drainage was instituted and maintained overnight and the patient then underwent surgery.

SURGERY Three days after the acute onset of symptoms, through a suboccipital craniectomy, a $3 \times 4 \mathrm{~cm}$ haematoma was evacuated from the medial aspect of the right cerebellar hemisphere. No clot was found in the fourth ventricle. Surgery was complicated by air embolism and cardiac arrest. No abnormal vessels were present in the surgical specimen.

POSTOPERATIVE COURSE In the immediate postoperative period the patient remained comatose but with a normal respiratory pattern. Oculocephalic and left corneal responses were present, but gag and right corneal responses were absent. Eye movements were disconjugate with equal non-reactive mid-position pupils. There was bilateral decerebrate posturing with a left hemiparesis and bilateral flexor plantar responses. By the third postoperative day, semipurposeful spontaneous movements were again evident and both pupils were reactive to light. By the ninth postoperative day she began opening her eyes and had conjugate eye movements. During this period she was intermittently febrile, had several episodes of gastrointestinal bleeding, and required daily lumbar punctures to decompress her bulging posterior fossa craniectomy site. A pneumoencephalogram done on the 22nd postoperative day demonstrated communicating hydrocephalus. Despite this, the patient continued to improve. By the 27 th postoperative day she responded to verbal commands, and by the 46th postoperative day she was verbalizing. At that time all cranial nerve function was intact although she continued to have hypotonia and ataxia of limbs and trunk. On the 93rd postoperative day a cisternal-pleural shunt was performed to control her hydrocephalus. After this she was able to progress to the rehabilitation ward where she showed continuing improvement in gait and speech. She is now in a special class for handicapped children. On the
WISC test, she has a verbal IQ score of 80 , although: she is considered to have a higher potential. She is? able to ambulate independently and perform alp activities of daily living. Cranial nerves are normal as are muscle strength and deep tendon reflexese Cerebellar signs are present in the upper and lower extremities bilaterally with greater involvement of the right side. These cerebellar abnormalities inter@ fere with function to a moderate degree.

DISCUSSION

Spontaneous cerebellar haematomas have usu $\underset{\text { के }}{\Rightarrow}$ ally been considered a problem of adults, and little attention has been given to their occurrence् in children. Hypertension, a problem seldom $\overline{\bar{n}}$. encountered in childhood, is the cause of the cerebellar haemorrhage in the majority of adultw cases.

By contrast, small angiomatous malformations are responsible for most cases of non-traumatic cerebellar haematomas in children (Hyland and Levy, 1954). Other aetiologies include large arteriovenous (A-V) malformations, sacculariw aneurysms, infection, and blood dyscrasias. A-d malformations of the posterior fossa may cause abnormal cerebellar or brain-stem function may cause subarachnoid haemorrhage, but awe rarely responsible for bleeding directly into $t \mathrm{Fe}_{\mathrm{c}}^{-}$ parenchyma of the cerebellum (Poppen, 1958; Verbiest, 1961). Ruptured intracranial aneurys

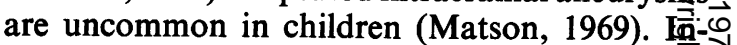
fection of the central nervous system, although! common in children, is an infrequent cause of intracranial haemorrhage (Dodge and Swartz, 1965). Blood dyscrasias also frequently involveō children and may cause intracranial bleeding, but bleeding elsewhere is usually present (Silverstein, 1961) and further investigation will confirm $\overrightarrow{\vec{B}}$ the presence of a systemic disease. Thus, in previously well children who have bleeding into the cerebellum of seemingly obscure aetiology, the responsible lesion has usually been a cerebellar angioma.

Angiomas as a cause of spontaneous cerebellar haematomas have been discussed in 8 several reviews (Hyland and Levy, 1954; Logue and Monckton, 1954; Crawford and Russell,으 1956; Odom, Tindall, and Dukes, 1961; Thrash, $D$ 1963). With one exception, these patients with angiomas were all under the age of 40 years, and five of the 23 patients in these reports were children 12 years of age or under. The term 'cryptic' has been proposed by Crawford and Russell, $\sigma$ (1956) to describe these angiomas, because the lesion is often difficult to identify due to its loca- $\frac{O}{2}$ 
TABLE 1

CEREBELLAR HAEMATOMAS IN CHILDREN DUE TO ANGIOMAS

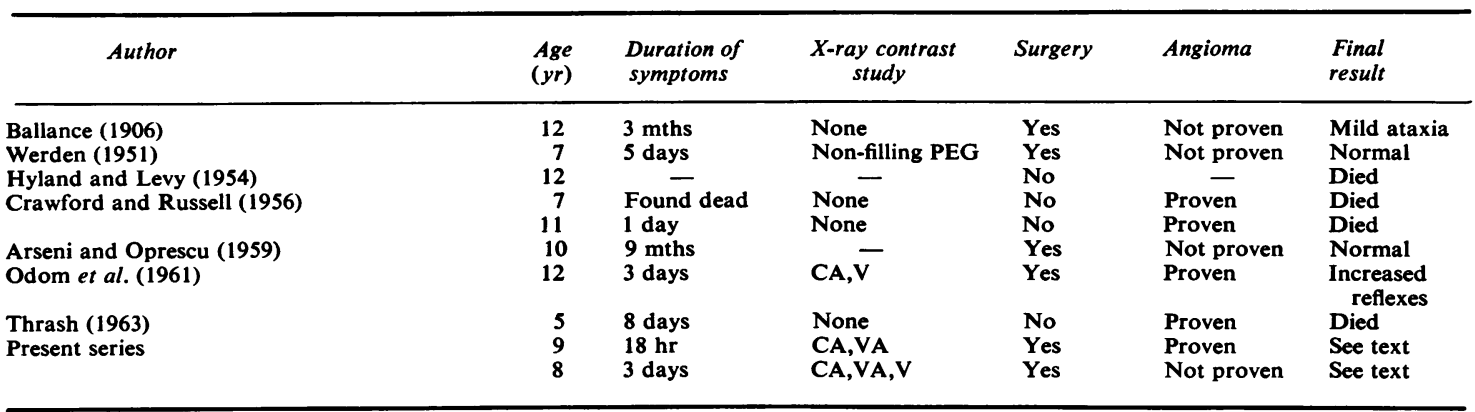

— information not given. PEG pneumoencephalogram. CA carotid angiogram. VA vertebral angiogram. V ventriculogram.

tion and small size. Most are situated deep in a cerebellar hemisphere, and enlargement and tortuosity of the vessels on adjacent brain surfaces are usually absent, making diagnosis difficult. Although the angioma is sometimes grossly visible in the wall of the haematoma, diagnosis is more often made as a result of microscopic examination of the surgical or necropsy specimen. These vascular lesions are composed of enlarged, tortuous, thin-walled arteries and veins. The small malformation may easily be destroyed by the massive haemorrhage that occurs (Crawford and Russell, 1956), making pathological identification impossible. Because of the difficulty in identifying such small lesions and because of the possibility of their destruction by the haemorrhage, most cases of unexplained spontaneous cerebellar haemorrhage in younger persons have been assumed to be caused by a ruptured angioma, even when no evidence of an angioma has been found.

Table 1 lists the 10 children reported in the English language medical literature who have had spontaneous cerebellar haematomas presumably due to angiomas. In six cases an angioma was actually found on examination of the tissue from surgery or necropsy, but in the other four cases an angioma was not proven even after pathological review of the specimen. Six of the 10 children survived after surgical intervention. The angioma was verified in only two of these survivors.

Table 2 reviews the clinical presentation and the course of the illness. The sequence of events in cerebellar haemorrhage in children and adults has been divided into four types: sudden death, acute onset leading to death within 48 hours;
TABLE 2

CLINICAL PICTURE ON ADMISSION

\begin{tabular}{llllll}
\hline \multicolumn{2}{c}{ Onset } & \multicolumn{2}{c}{ State of consciousness } & \multicolumn{2}{c}{ Signs } \\
\hline Acute & 7 & Dead on arrival & 1 & Ataxia & $4 / 7$ \\
Chronic & 2 & Awake & 1 & Nuchal rigidity & $3 / 7$ \\
Unknown & 1 & Drowsy & 4 & Nystagmus & $3 / 7$ \\
\multicolumn{2}{c}{ Symptoms } & Coma & 3 & Facial weakness & $2 / 7$ \\
Headache & $8 / 8$ & Unknown & 1 & Papilloedema & $1 / 7$ \\
Nausea and & & & & Hemiparesis & $1 / 7$ \\
vomiting & $8 / 8$ & & & Ocular bobbing & $1 / 7$ \\
Seizure & $2 / 8$ & & & Unequal pupils & $1 / 7$ \\
\hline
\end{tabular}

survival beyond 48 hours with late deterioration; and spontaneous resolution (Lichtenstein, 1968).

One of the 10 children represents an example of sudden death (Crawford and Russell, 1956). Case 1 in our study would almost certainly have fallen into this same category had there been any significant delay before his arrival at the hospital.

Acute onset leading to death within 48 hours is the most common clinical course seen in adults (Mitchell and Angrist, 1942; Hyland and Levy, 1954; Rey-Bellet, 1960) occurring in $64 \%$ of patients in Dinsdale's series (1964). Only one of the ten children, however, followed this course (Crawford and Russell, 1956).

Two-thirds of the children fell into the category of patients who survived more than 48 hours without treatment. These patients can be subdivided into two groups. One group consists of those patients having an acute onset but slower deterioration, with death or successful surgical intervention occurring three to 10 days after onset of illness. The second group within this category consists of patients whose symptoms and signs are those of a slowly expanding cerebellar mass evolving over a period of several 
weeks or months. Cerebellar findings are almost always present along with findings of increased intracranial pressure.

Spontaneous resolution has not been recorded in children, although it cannot be stated definitely whether or not this occurs. It has been documented as occurring in adults (Mitchell and Angrist, 1942; Norris et al., 1969) but is the least common type of clinical course.

The ten children listed in Table 1 had all been in good health before their cerebellar haemorrhage. After the haemorrhage, headache and vomiting were the only consistent findings (Table 2). One child was awake on admission while four were drowsy, three were in coma, and one had died before being admitted to the hospital. In one patient, the state of consciousness was not described.

Nystagmus or ataxia was found in four of the five children in whom this could be tested. Peripheral facial weakness was reported in two cases and ocular bobbing in one case. Both findings have previously been reported in cerebellar haemorrhage (Fisher, Picard, Polak, Dalal, and Ojemann, 1965). Papilloedema was reported in only one case. One patient had unequal pupils. Although most patients have small but equal and reactive pupils, one-third may have asymmetry of pupillary size (Dinsdale, 1964).

Findings usually associated with disease of the cerebral hemispheres were present in three children. Two children had generalized seizures shortly after the onset of symptoms. This has been seen in both children (Crawford and Russell, 1956) and adults (Mitchell and Angrist, 1942) with cerebellar haematomas. A unilateral hemiparesis, as found in one child, has also been previously noted (Hyland and Levy, 1954; Fisher et al., 1965).

In the five children who were described as awake or drowsy at the time of admission, a rather clearly defined picture was present. This included a previously well child with the acute onset of headache and vomiting, decrease in the level of consciousness, and the presence of nystagmus and ataxia. In children admitted in coma, diagnosis is difficult since cerebellar signs cannot be elicited. Each of the three children who were comatose upon admission, however, had a history of the acute onset of headache and vomiting preceding the rapid onset of their coma. The history alone was strongly suggestive of intracranial haemorrhage, and this was confirmed in the two cases where lumbar puncture was performed. As in the two cases in the present $\stackrel{\mathbb{D}}{=}$ report, subarachnoid haemorrhage with rapid $\underline{0}$ deterioration is always an indication for immedi- $z$ ate investigation of the possibility of an intra- $\frac{c}{\circ}$ cranial vascular malformation and/or haema- of toma.

The safety of performing lumbar punctures in ${ }_{0}^{0}$ patients suspected of having a cerebellar haematoma is a matter of some controversy. Downward tonsillar herniation after spinal tap has been $\vec{z}$

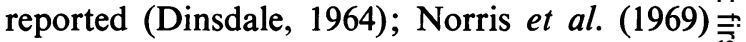
therefore suggest that this procedure be withheld. Others report that this relationship is unclear and $\frac{\text { 음 }}{0}$ that performing a lumbar puncture is a risk that $\frac{\bar{D}}{\bar{D}}$. must be taken to confirm the diagnosis (Fisher, $\overparen{\Phi}$ et al., 1965). The cerebrospinal fluid (CSF) find- $\frac{\circ}{2}$ ings are not always as expected. Although usu- $\infty$ ally bloody or xanthochromic and under in- $\vec{\circ}$ creased pressure, the CSF in one child (Werden, $\vec{\omega}$ 1951) was found to be clear and colourless and $\stackrel{\circ}{\circ}$ under normal pressure. One possible explanation for this occurrence is the rapidity with which lumbar puncture is performed after the onset of $o$ symptoms. Insufficient time may have elapse $\omega$ for the extravasated blood to reach the lumb subarachnoid space (Fisher et al., 1965), and repeat spinal tap may show blood in the CSW (Giroux and Leger, 1962). Nuchal rigidity was present in three children but absent in four cases. Nuchal rigidity has been found to be absent 站 $\overrightarrow{0}$ the majority of cases involving adults (Re N Bellet, 1960; Lichtenstein, 1968).

Radiological contrast studies had been described in only two children before the two children in the present report. In one of these two previous children, a non-filling pneumo- $\stackrel{\mathbb{Q}}{\complement}$ encephalogram had been performed. All three $\overrightarrow{\overrightarrow{0}}$ children with completed studies had carotid 3 angiograms which revealed a wide sweep of the anterior cerebral artery indicating the presence of hydrocephalus. Vertebral angiography was performed in two children and was abnormal in both. An avascular area was present in both cases and, in addition, one also showed abnormal vessels. Ventriculograms were done in two child- $\frac{\circ}{3}$ ren and showed enlargement of the lateral and 0 third ventricles with anterior displacement of the aqueduct.

Although each of the contrast studies performed in children was abnormal, such success has not always been reported in adults. McKis- $N$ sock, Richardson, and Walsh (1960) interpreted $N_{\omega}$ the ventriculograms as normal in $25 \%$ of patients subsequently proven to have cerebellar haemato- 
mas; five of the six vertebral angiograms were also interpreted as normal. It is not known whether this is related to a real difference between children and adults, to the small number of studies that have been performed in children, or to improved diagnostic abilities. In our view the initial study for children admitted with a clinical picture compatible with the presence of a vascular lesion should be a transfemoral catheter study of the intracranial circulation. If vertebral angiography is not diagnostic, carotid angiography can then be pursued. If necessary, diagnosis or confirmation of the diagnosis can then be made by ventriculography.

Six of the 10 children were operated upon, and all survived after surgical removal of the haematoma. The remaining four children were not operated upon and succumbed. Among adults, in whom cerebellar haematomas are much commoner, only 40 cases of successful surgery have been reported in the English language medical literature. These successes have almost always been in adults who survived more than 48 hours or presented with long-standing symptoms. Lichtenstein reviewed this literature up to 1968. He cited reports of 28 successful cases and added two cases of his own. Two other cases were reported before 1968 (Logue and Monckton, 1954), but were not included in his review, and the remaining eight cases were contained in more recent publications (Norris et al., 1969; Chawla, 1970).

Thus it would seem that children with cerebellar haematomas have a better prognosis than do adults. One possibility for this relatively high survival rate is that there has been incomplete case reporting, with the majority of cases in children being found among reports of surgical successes. This seems unlikely, since no children with non-traumatic cerebellar haematomas were found among the more than 40,000 necropsies reported in three large necropsy series (Michael, 1932; Rey-Bellet, 1960; Dinsdale, 1964). The apparently more favourable prognosis may be related to several factors. All the children had enjoyed good health before their cerebellar haemorrhage and did not have chronic underlying disease such as hypertension. Although the onset of their illness may have been acute, the majority of the childhood cases were not comatose on admission, and they were able, without surgical treatment, to survive the critical first 48 hours during which time most adult patients die. In these noncomatose patients, the majority of the symptoms and neurological signs pointed to an abnormality in the posterior fossa. In the comatose patients, a picture compatible with intracranial haemorrhage indicated the need for rapid intervention.

The two cases reported here raise several important points. They emphasize that spontaneous cerebellar haematomas do occur in children and are reversible lesions irrespective of the state of consciousness on admission. The first case was admitted in coma with agonal respirations and absent brain-stem function. The second case was also admitted in coma and had irregular respiration, bilateral decerebrate posturing, bilateral Babinski signs, and a single dilated pupil. Both cases are representative of the group of patients with acute onset who, without surgical intervention, continue to deteriorate and expire within the ensuing 48 hours. Irregular respiration (Hyland and Levy, 1954), a unilateral dilated pupil (Fisher et al., 1965), decerebrate posturing (Norris et al., 1969), Babinski signs (Fisher et al., 1965), and depressed state of consciousness have each been assumed by many authors to indicate a state where spontaneous recovery could no longer be expected, at least in adults. The most important prognostic sign has been the state of consciousness. Before this report, no patient admitted in coma had survived, and only three patients, all adults, had been successfully operated upon within the first 48 hours after the acute onset of symptoms. It has even been suggested that diagnostic procedures in these comatose patients were not urgent since little hope of survival could be offered (Plum and Posner, 1966). Although not in coma upon admission, some adult patients have deteriorated to a comatose state but made good recoveries after surgery.

Several interesting events marked the postoperative course in our patients. The first child exhibited akinetic mutism for five months after surgery. Mutism has been noted in several adults with cerebellar haematomas, occurring shortly after the acute onset of the illness (Giroux and Leger, 1962; Dinsdale, 1964; Fisher et al., 1965). One patient had akinetic mutism during the four month period between the time of surgery and his subsequent death (Fisher et al., 1965). Although the clinical appearance of this state had been well documented, no clear correlation with the pathological material could be made in Dinsdale's six patients who came to necropsy and who had been mute before death (Dinsdale, 1964). Akinetic mutism has been found to be 
related to the presence of hydrocephalus (Messert, Henke, and Langheim, 1966), but in our patient the resolution of the akinetic mutism does not seem to be temporally related to the resolution of the hydrocephalus.

Persistent hydrocephalus developed in both children. The initial indication of abnormal CSF dynamics was fullness at the suboccipital operative site with the formation of a pseudomeningocoele. The hydrocephalus was treated with cerebrospinal fluid shunting procedures. Persistent hydrocephalus in patients with cerebellar haemorrhage is uncommon and has been mentioned only twice before. Schreiber (1963) described a newborn infant who was successfully operated upon for a traumatic cerebellar haematoma, but subsequently required a ventriculojugular shunt for relief of hydrocephalus. In addition, there has been a report of one adult patient who survived without surgery but required a ventriculoatrial shunt (Norris et al., 1969).

Those patients who survive after surgery have a good prognosis for functional recovery. The majority of adult patients have either had normal neurological examinations or had mild residual cerebellar abnormalities on follow-up testing. Of the four surviving children mentioned in previous reports, two were subsequently normal, one had a mild ataxia, and one had persistently increased deep tendon reflexes on one side. In all these patients, return of function and stabilization of the neurological examination occurred within several months after surgery.

Our two patients have not done as well in that they still show moderately severe bilateral ataxia and persistence of abnormal pyramidal tract and cranial nerve signs. The ultimate degree of recovery, however, has not yet been reached, and they both continue to show steady improvement despite the lapse of many months since the time of surgery. In previous cases, the time interval between surgery and subsequent stabilization of neurological function has been much shorter. This is most likely due to the fact that none of the previous survivors had been so severely depressed or in such poor neurological condition at the time of admission.

\section{REFERENCES}

Arseni, C., and Oprescu, I. (1959). Cerebellar hematomas. Journal of Neurosurgery, 16, 503-507.

Ballance, H. A. (1906). Case of traumatic haemorrhage into the left lateral lobe of the cerebellum, treated by operation, with recovery. Surgery, Gynecology and Obstetrics, 3, 223 225.

Chawla, J. C. (1970). Spontaneous intracerebellar haemor rhage. British Medical Journal, 1, 93-94.

Crawford, J. V., and Russell, D. S. (1956). Cryptic arterio venous and venous hamartomas of the brain. Journal of Neurology, Neurosurgery, and Psychiatry, 19, 1-11.

Dinsdale, H. B. (1964). Spontaneous haemorrhage in the posterior fossa. Archives of Neurology, 10, 200-217.

Dodge, P. R., and Swartz, M. N. (1965). Bacterial menin gitis-a review of selected aspects. New England Journal of Medicine, 272, 1003-1010.

Fang, H. C. H., and Foley, J. M. (1954). Hypertensive haemorrhages of the pons and cerebellum. Archives of: Neurology and Psychiatry, 72, 638-639.

Fisher, C. M., Picard, E. H., Polak, A., Dalal, P., and Ojemann, R. G. (1965). Acute hypertensive cerebella? haemorrhage: diagnosis and surgical treatment, Journal of Nervous and Mental Disease, 140, 38-57.

Giroux, J. C., and Leger, J. L. (1962). Haematomas of the posterior cranial fossa: a report of three cases. Canadian? Medical Association Journal, 87, 59-61.

Hyland, H. H., and Levy, D. (1954). Spontaneous cerebellar haemorrhage. Canadian Medical Association Journal,, 71, 315-323.

Lichtenstein, R. S. (1968). Spontaneous cerebellar hema- $\vec{\omega}$ tomas. A report of three operated cases and review of the literature. Johns Hopkins Medical Journal, 122, 319-328.

Logue, V., and Monckton, G. (1954). Posterior fosså angiomas. A clinical presentation of nine cases. Brain, 77 w్

McKissock, W., Richardson, A., and Walsh, L. (1968) Spontaneous cerebellar haemorrhage. A study of 34 cథi secutive cases treated surgically. Brain, 83, 1-9.

Matson, D. D. (1969). Neurosurgery of Infancy and Ch $\stackrel{\mathbb{R}}{d}$ 음 hood, 2nd edn., p. 934. Thomas: Springfield, Ill.

Messert, B., Henke, T. K., and Langheim, W. (196/). Syndrome of akinetic mutism associated with obstructêf hydrocephalus. Neurology (Minneap.), 16, 635-649.

Michael, J. C. (1932). Cerebellar apoplexy. Americ్ț Journal of Medical Sciences, 183, 687-695.

Mitchell, N., and Angrist, A. (1942). Spontaneous cere $N$ bellar hemorrhage. American Journal of Pathology, 18. 935-954.

Norris, J. W., Eisen, A. A., and Branch, C. L. (1969) Problems in cerebellar haemorrhage and infarction Neurology (Minneap.), 19, 1043-1050.

Odom, G. L., Tindall, G. T., and Dukes, H. T. (1961) Cerebellar hematoma caused by angiomatous malformations. Report of four cases. Journal of Neurosurgery, $18, \underline{\underline{B}}$ 777-782.

Plum, F., and Posner, J. B. (1966). The Diagnosis of Stupor? and Coma, pp. 97-100. Davis: Philadelphia, Pa.

Poppen, J. L. (1959). Clinical Neurosurgery, p. 289. Williams \& Wilkins: Baltimore, Md.

Rey-Bellet, J. (1960). Cerebellar hemorrhage. A clinico pathologic study. Neurology (Minneap.), 10, 217-222.

Schreiber, M. S. (1963). Posterior fossa (cerebellar) haematoma in the new-born. Medical Journal of Australia 2, 713-715.

Silverstein, A. (1961). Intracranial hemorrhage in patients with bleeding tendencies. Neurology (Minneap.), 11, 310-0 317.

Thrash, A. M. (1963). Vascular malformations of the cere $D$ bellum. Report of four fatal cases. Archives of Pathology 75, 65-69.

Verbiest, H. (1961). Arterio-venous aneurysms of the posterior fossa, analysis of six cases. Acta Neurochirurgica $\mathrm{N}$ 9, 171-195.

Werden, D. H. (1951). Spontaneous intracerebral and N్W cerebellar hematoma. Report of six cases with operative treatment. Bulletin of the Los Angeles Neurological Society, 16, 174-184. 